\title{
Hemodynamic Effect of Unequal Anterior Cerebral Artery Flow Rates on the Anterior Communicating Artery Bifurcation: A Computational Fluid Dynamics Study
}

\author{
Thomas Rau, ${ }^{1}$ Xing He, ${ }^{2}$ Prem Venugopal, ${ }^{2}$ Fernando Viñuela, \\ Gary Duckwiler, ${ }^{2}$ and Daniel J. Valentino ${ }^{2}$ \\ ${ }^{1}$ Department of Radiology, University of Colorado Health Sciences Center, Aurora, CO 80045, USA \\ ${ }^{2}$ Department of Radiological Sciences, University of California, Los Angeles, CA 90095, USA \\ Correspondence should be addressed to Thomas Rau, thomas.rau@ucdenver.edu
}

Received 12 November 2007; Revised 11 June 2008; Accepted 11 December 2008

Recommended by Ewa Pietka

\begin{abstract}
Computational fluid dynamics techniques were used to investigate the hemodynamic effect of unequal anterior cerebral artery flow rates on the anterior cerebral and anterior communicating artery (ACA-ACOM) bifurcation. Hemodynamics have long been implicated as a major factor in cerebrovascular disease. Using an idealized 2D symmetric model of the ACA-ACOM geometry, the flow field and wall shear stress (WSS) at the bifurcation regions are assessed for pulsatile inflows with left to right flow ratios of $1: 1,2: 1,3: 1$, and $4: 1$. Unequal flow rates through the ACA parent arteries result in bifurcation of the higher flow parent stream and a shifting of the impingement points along the A2-ACOM adjoining wall toward the contralateral ACA. Cross-flow through the ACOM is generally unstable and results in increased WSS at the impingement region from the higher flow parent artery and a double amplitude peak in the WSS at the contralateral bifurcation region from local recirculation effects. These results suggest that asymmetry in ACA flow rates result in increased hemodynamic stresses at the ACA-ACOM bifurcation regions and suggest a possible factor for vessel weakening and aneurysm formation.
\end{abstract}

Copyright (C) 2008 Thomas Rau et al. This is an open access article distributed under the Creative Commons Attribution License, which permits unrestricted use, distribution, and reproduction in any medium, provided the original work is properly cited.

\section{Introduction}

Numerous investigations on the hemodynamics in the Circle of Willis have been performed to elucidate the pathogenesis of aneurysm formation. It is known that over $85 \%$ of aneurysms occur in the anterior circulation and form predominantly at the apex of vessel bifurcations and around sharp bends. It is at these locations where hemodynamic stresses are the greatest and the structural protein assembly of the muscular layer of the vessel wall is most underdeveloped [1]. A generally accepted theory is that an anomalous response of the vascular endothelium to oscillating wall shear stresses leads to progressive and unstable degradation of the arterial wall and consequent aneurysm formation [2]. The validity and reliability of computational methods to model the hemodynamic processes has motivated numerous computational flow studies to characterize the flow patterns and wall shear stresses associated with pulsatile flows in these susceptible regions [3-9].
The junction of the anterior cerebral (ACA) and anterior communicating (ACOM) arteries represents a unique and complex vascular geometry with two closely apposed bifurcations (see Figure 1). Studies in the past have shown that a strong association exists between the development of aneurysms and their neck sizes at the ACOM junction with the dominant A1 parent artery feeder [10]. In this study, a computational fluid dynamics (CFD) simulation was performed on an idealized $2 \mathrm{D}$ symmetric model of the ACA and ACOM bifurcation to investigate the hemodynamic effect of unequal flow rates through the A1 ACA segments. Simulations were run with left to right inflow ratios fixed at $1: 1,2: 1,3: 1$, and $4: 1$. The overall flow patterns as well as the wall shear stress (WSS) at various points along the adjoining wall between the A2 and ACOM segments are analyzed spatially and temporally near the impingement points where aneurysms are predicted to occur. Unequal leftright A1 flow rates can occur whenever the total upstream flow resistances are not equal bilaterally. Clinically, this may 


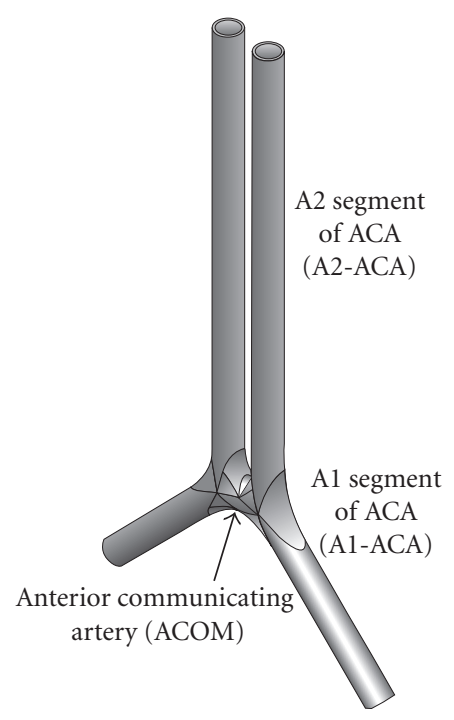

FIGURE 1: A 3D model depiction of the anterior cerebral circulation upon which the simulation is based.

occur with unilateral high grade stenotic lesions in upstream vessels to the Circle of Willis. This might be seen after a thromboembolic event in stroke victims and succeeding vasospasm in upstream arteries or the existence of a congenital hypoplastic A1-ACA. Asymmetric flow rates between the A1 segments can also occur transiently by iatrogenic means during a rapid intravenous infusion of contrast media during diagnostic cerebral angiography. This study attempts to characterize some hemodynamic stress pattern differences which may occur under these circumstances.

\section{Methods}

A 2D symmetric model of the ACA and ACOM was constructed using the Solidworks [11] software seen in Figure 2. The diameter was assigned a unit length of 1 , and all other dimensions were scaled accordingly and proportioned from other studies $[12,13]$. The bifurcation angle from the A1 to A2 segments of the anterior cerebral arteries was chosen to be 45 degrees, within the range of clinical models. The radii of curvature adjoining the walls of the A1 to the A2 and ACOM segments were chosen such that a smooth transition was made. The model was then imported into FLUENT's Gambit for meshing. A finer-graded mesh was used at the boundary layer along all wall surfaces to increase the resolution of the gradients near the wall. A total of 196944 data points were obtained after meshing.

Substitution of the following nondimensional expressions for velocity $u$ and $v$, length $x$ and $y$, time $t$, pressure $P$, and Reynolds number Re allows us to undimensionalize the governing equations of continuity and Navier-Stokes in the Cartesian coordinate system. We divide the flow variables with the corresponding references variables denoted by subscript ref to produce the nondimensional variables denoted by $*$. Let $\rho$ be the density of blood, $\mu$ the dynamic viscosity of blood, $u_{\text {ref }}$ be the reference velocity, and $L_{\text {ref }}$ the reference length, where

$$
\begin{gathered}
u^{*}=\frac{u}{u_{\mathrm{ref}}}, \quad v^{*}=\frac{v}{u_{\mathrm{ref}}}, \\
x^{*}=\frac{x}{L_{\mathrm{ref}}}, \quad y^{*}=\frac{y}{L_{\mathrm{ref}}}, \\
t^{*}=\frac{t u_{\mathrm{ref}}}{L_{\mathrm{ref}}}, \quad P^{*}=\frac{P}{\rho u_{\mathrm{ref}}^{2}}, \\
\operatorname{Re}=\frac{\rho u_{\mathrm{ref}} L_{\mathrm{ref}}}{\mu} .
\end{gathered}
$$
[14]

The equations of continuity and Navier-Stokes become

$$
\begin{aligned}
& \frac{\partial u^{*}}{\partial x^{*}}+\frac{\partial v^{*}}{\partial y^{*}}=0, \\
& \frac{\partial u^{*}}{\partial t^{*}}+u^{*} \frac{\partial u^{*}}{\partial x^{*}}+v^{*} \frac{\partial u^{*}}{\partial y^{*}}=-\frac{\partial p^{*}}{\partial x^{*}}+\frac{1}{\operatorname{Re}}\left(\frac{\partial^{2} u^{*}}{\partial x^{* 2}}+\frac{\partial^{2} u^{*}}{\partial y^{* 2}}\right), \\
& \frac{\partial v^{*}}{\partial t^{*}}+u^{*} \frac{\partial v^{*}}{\partial x^{*}}+v^{*} \frac{\partial v^{*}}{\partial y^{*}}=-\frac{\partial p^{*}}{\partial y^{*}}+\frac{1}{\operatorname{Re}}\left(\frac{\partial^{2} v^{*}}{\partial x^{* 2}}+\frac{\partial^{2} v^{*}}{\partial y^{* 2}}\right) .
\end{aligned}
$$

The usage of the governing flow equations in this form eliminates dependence on absolute dimensions and makes the simulation dependent only on the Reynold's number. The results obtained can, therefore, be more generalized. Incompressible, Newtonian fluids were assumed in the calculations of the nondimensional Navier-Stokes equations, and the solutions were performed using OpenFOAM software [15]. A minimum Reynold's number of 400 was set by allowing fluid density to be equal 1 , scaling the maximum of the velocity profile to 1 , and setting fluid viscosity to 0.0025 . The Reynold's number was allowed to vary up to 800 by the nature of the pulsatile velocity profile at the 2 inlets. Two-plug flow inlets were assigned at the parent anterior cerebral arteries with the pulse arterial waveform defined by (3). A no-slip and nonelastic condition was imposed on the walls and the outflow boundary was set at a reference pressure of 0 . The simulations were allowed to run for three to four complete cycles at 60 beats per minute to achieve convergence of solutions.

Simulations were run with the ratio of the inflow rates between the left and right A1 segments set at $1: 1,2: 1$, $3: 1$, and $4: 1$. This was conducted by decreasing the timedependent amplitude of the right A1 inflow segment by the appropriate fraction $(0.5,0.33$, and 0.25$)$.

The velocity, $U$, pulse profile used in these simulations was given by

$$
\begin{array}{rl}
U(t)=8 & .419 \\
* & \{0.08225 \\
& +2(-0.013077 \cos (w t)-0.002868 \sin (w t)) \\
& +2(0.001125 \cos (2 w t)-0.001625 \sin (2 w t)) \\
& +2(-0.000172 \cos (3 w t)+0.001631 \sin (3 w t)) \\
& +2(-0.001000 \cos (4 w t))\},
\end{array}
$$

where $w=2 \pi / T, T=1$ second. 


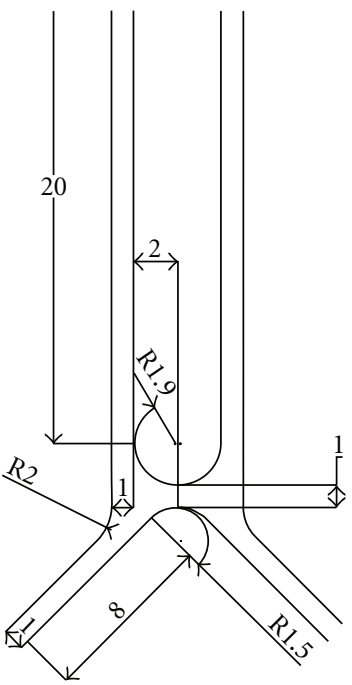

(a)

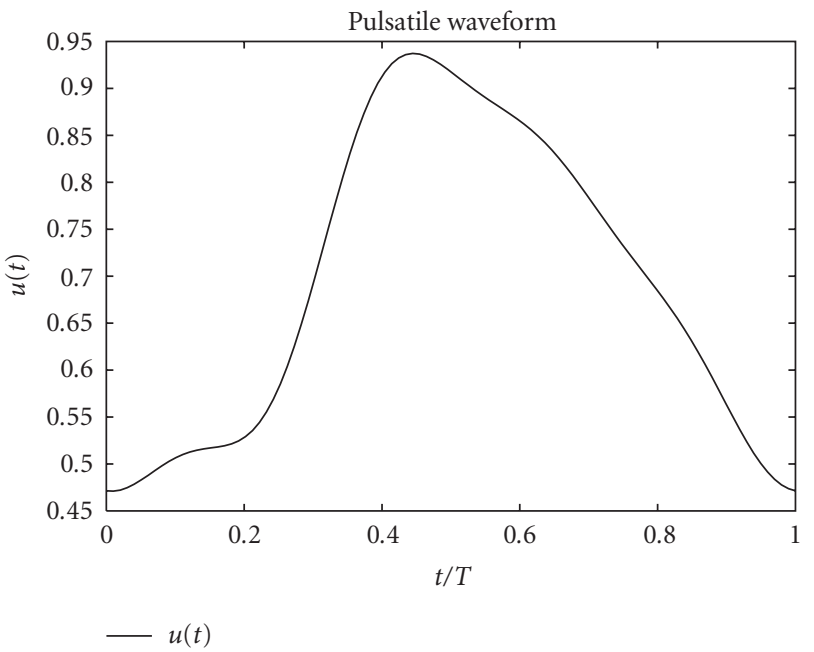

(b)

Figure 2: (a) ACA-ACOM geometry with 45 degree bifurcation angles. (b) Pulse waveform given by (3). The global maximum occurring at $0.45 t / T$ will define the transition between the systolic and diastolic phases of the flow waveform.

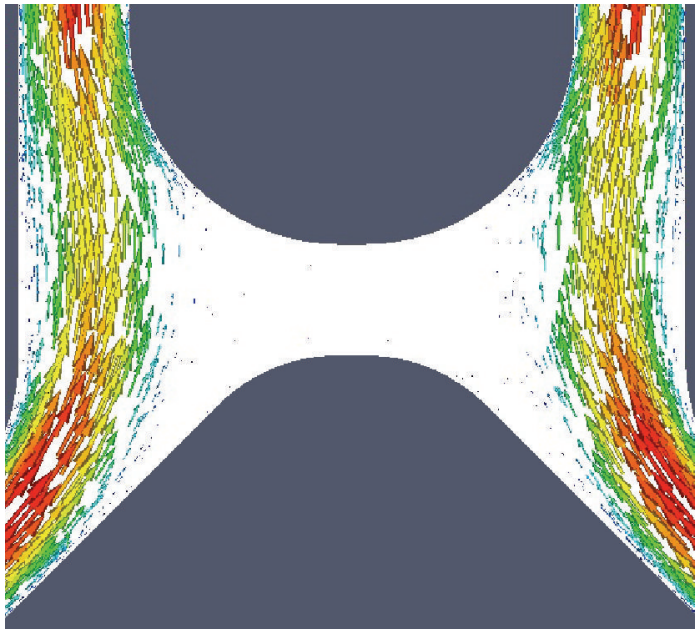

Vol point interpolate $(U)$ magnitude $\begin{array}{lllll}0 & 0.373 & 0.747 & 1.12 & 1.49\end{array}$

(a)

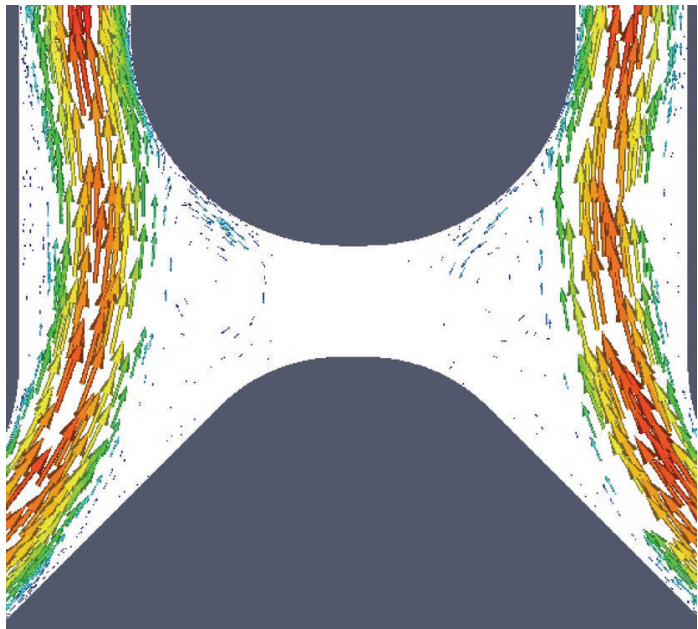

Vol point interpolate $(U)$ magnitude

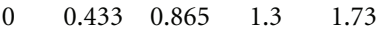

(b)

Figure 3: Velocity field with equal left and right ACA flow rates $\left(Q_{R}=Q_{L}\right)$ during (a) systole and (b) early diastole. A dead zone in the ACOM exists throughout the pulsation cycle. (b) Unsteadiness exists bilaterally at the A2-ACOM impingement sites with flow separation and vortices seen during the diastolic phase.

The transition point between the systolic and diastolic phases of the velocity wave form will be defined at the global maximum occurring at $0.45 \mathrm{t} / \mathrm{T}$.

The wall shear stress (WSS) is obtained with the following equation:

$$
\left(\begin{array}{l}
W_{x} \\
W_{y}
\end{array}\right)=\mu\left(\begin{array}{cc}
2 \frac{\partial u}{\partial x} & \frac{\partial u}{\partial y}+\frac{\partial v}{\partial x} \\
\frac{\partial u}{\partial y}+\frac{\partial v}{\partial x} & 2 \frac{\partial v}{\partial y}
\end{array}\right)\left(\begin{array}{l}
n_{x} \\
n_{y}
\end{array}\right)
$$

where $n_{x}$ and $n_{y}$ are the $x$ and $y$ components of the normal vector of vessel wall.

\section{Results}

The relative inflow rates through the two A1 parent artery segments greatly affect the overall flow pattern and the hemodynamic stresses on the walls at the bifurcation region. When the left-right A1 inflow rates are unequal, bifurcation of the higher velocity parent stream occurs and the ACOM 


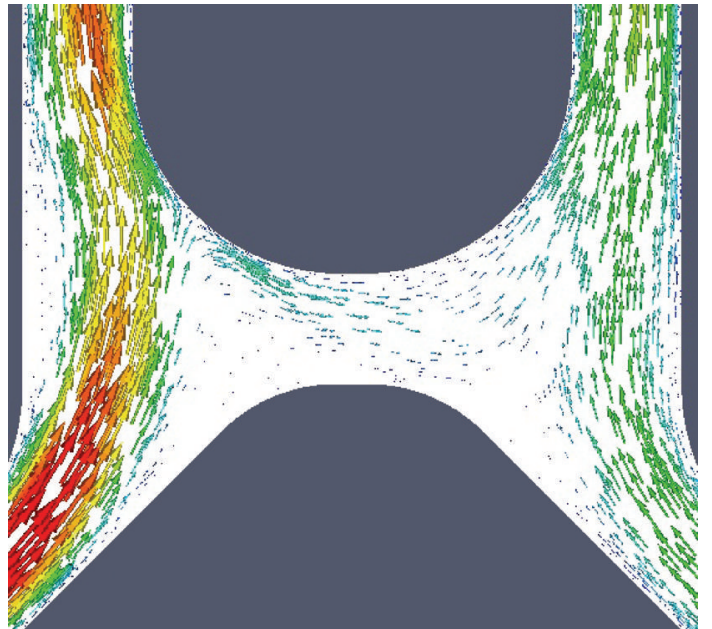

Vol point interpolate $(U)$ magnitude

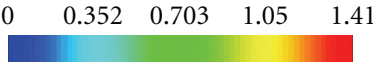

(a)

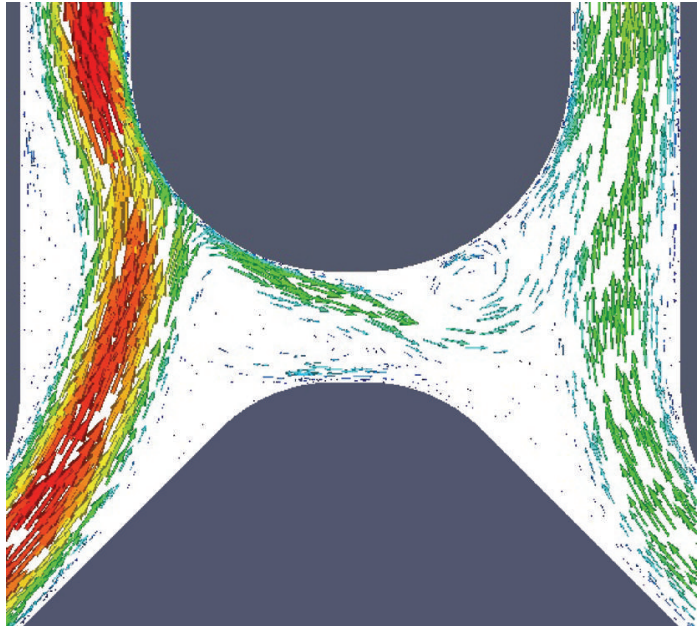

Vol point interpolate $(U)$ magnitude $\begin{array}{lllll}0 & 0.407 & 0.814 & 1.22 & 1.63\end{array}$

(b)

FIgURE 4: (a) Velocity field during systole and (b) early diastole with ACA flow rates set at $Q_{\mathrm{R}}=0.5 Q_{\mathrm{L}}$. Left ACA impingement site moves rightward along the A2-ACOM adjoining wall as compared to the case where $Q_{R}=Q_{L}$ with bifurcation of flow into the A2-ACA and ACOM segments. (b) A large vortex is generated on the right bifurcation site during diastole.

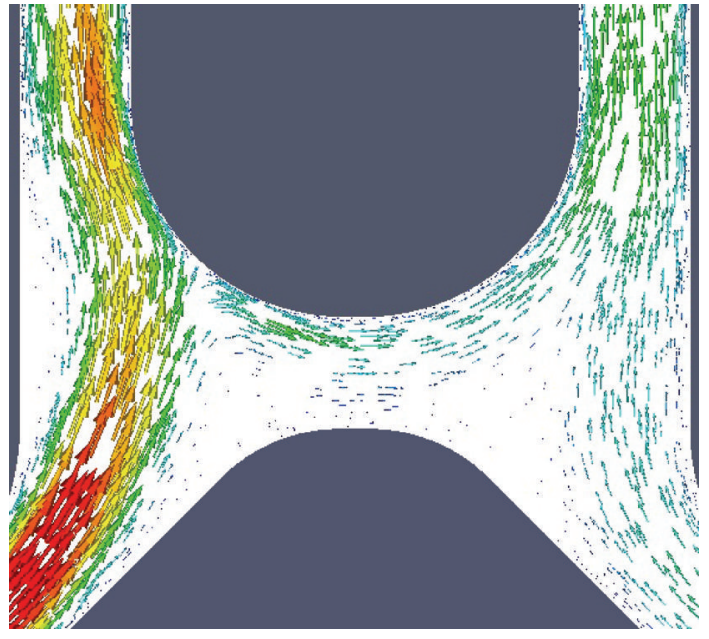

Vol point interpolate $(U)$ magnitude $\begin{array}{lllll}0 & 0.351 & 0.701 & 1.05 & 1.4\end{array}$

(a)

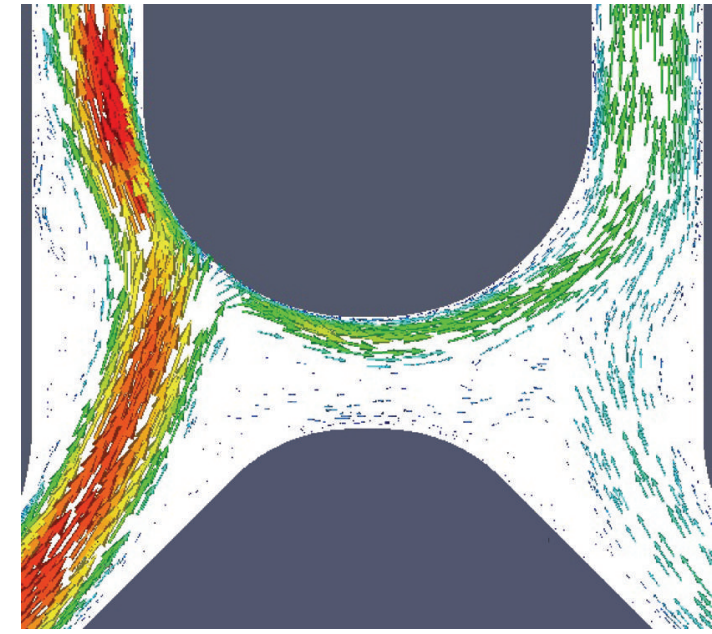

Vol point interpolate $(U)$ magnitude $\begin{array}{lllll}0 & 0.413 & 0.827 & 1.24 & 1.65\end{array}$

(b)

Figure 5: (a) Velocity field during systole and (b) early diastole with ACA flow rates set at $Q_{\mathrm{R}}=0.33 Q_{\mathrm{L}}$. Again bifurcation of the A1 parent flow artery into the ACOM and A2 segments is seen. Left ACA impingement site moves further rightward along A2-ACOM adjoining wall compared to that seen in the case where $Q_{\mathrm{R}}=0.5 Q_{\mathrm{L}}$. Flow separation is again seen on the right $\mathrm{A} 2$-ACOM impingement site leading to local recirculation effects.

becomes a functional anastomosis. While flow through the A1 and A2 ACA segments is generally laminar, unsteadiness is seen in the ACA-ACOM bifurcation section especially during the deceleration phase of pulsation. Figures 4, 5, and 6 show the bifurcation of the higher flow parent artery streams. The flow through the junction appears more stabilized during the acceleration phase of the pulse cycle.
For equal left-right A1 flow rates, separation and vortex shedding occur at the ACOM junction as the streams bypass the anastomosis into the A2 segment. Thus, while the ACOM is rendered a dead zone, a local unsteady region is created near the impingement site resulting in irregular, low amplitude WSS patterns at the A2-ACOM adjoining wall. Figure 3 shows the bilateral oblique impingement zones of 


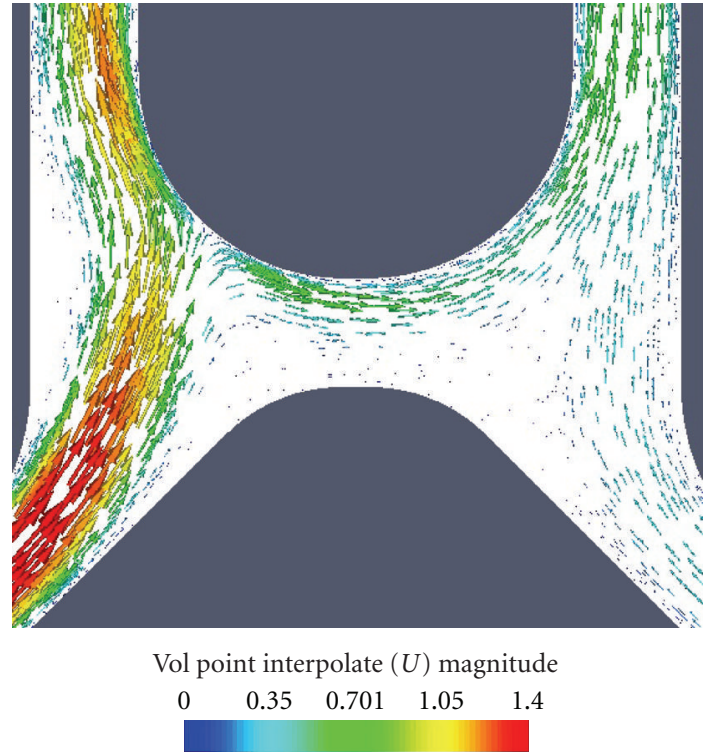

(a)

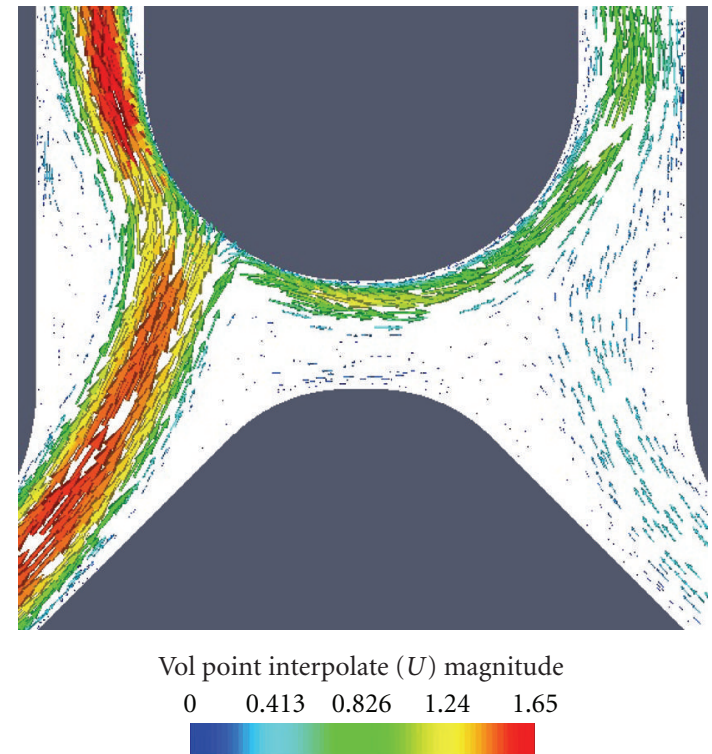

(b)

Figure 6: (a) Velocity field during systole and (b) early diastole with ACA flow rates set at $Q_{\mathrm{R}}=0.25 Q_{\mathrm{L}}$. Bifurcation of the A1 parent flow artery into the ACOM and A2 segments is again seen. Left ACA impingement site appears to move even further rightward along A2-ACOM adjoining wall compared to that seen in the case where $Q_{R}=0.33 Q_{L}$. Flow separation is again seen on the right A2-ACOM impingement site leading to local recirculation effects.

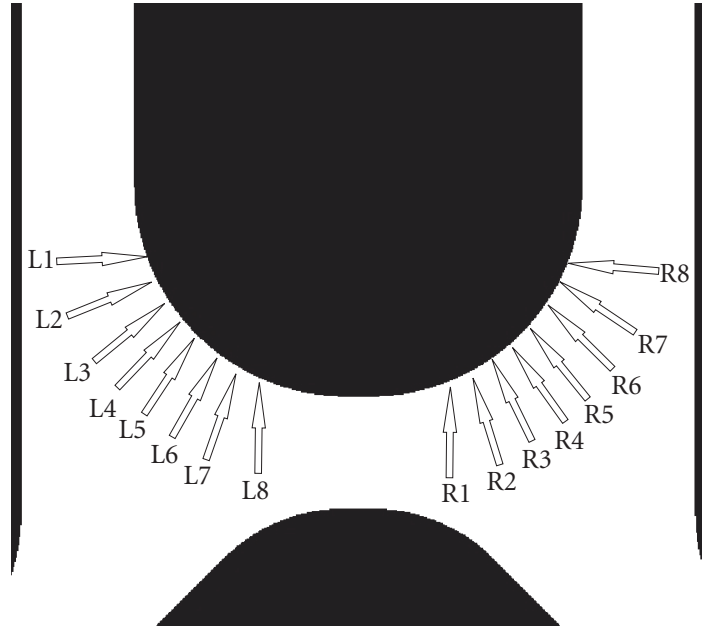

FIGURE 7: Labeled points on the left and the right impingement points in the WSS plots.

the parent A1 segments onto the distal A2-ACOM adjoining wall. The WSS plots in Figures 8 and 12 demonstrate smaller amplitude WSS and gradients compared to the unequal ACA flow rate cases.

As the inflow rate from the right ACA is reduced relatively to the left, the impingement point of the left A1 stream moves toward the contralateral bifurcation along the A2-ACOM adjoining wall segment and is divided. The impingement point is also observed to oscillate toward the contralateral ACA segment during the deceleration phase of the pulsation cycle. The majority of the inflow continues into the A2 segment while the divided fraction enters the ACOM segment and joins the flow through the right ACA. Flow through the ACOM anastomosis is subject to separation as it negotiates the curvature of the ACOM during the deceleration phase of the pulsation cycle. The flow separation results in a large second peak in time in the WSS plots due to local recirculation effects. The recirculation zone is most apparent in the case where $Q_{\mathrm{R}}=0.5 Q_{\mathrm{L}}$ in Figure $4(\mathrm{~b})$ but can be seen in the other cases at high magnification views (not shown). The magnitude of the second peak appears to increase as the ratio of the left to right flow rates is increased and occurs earlier in the pulsation cycle. Thus, unequal A1 flow rates impose additional stresses on the ACA-ACOM bifurcation region by the degree of unbalanced left-right A1 inflow rates.

The plots of WSS versus time and position are shown in Figures 8, 9, 10, and 11 for the various left to right flow ratios. The positions along the left and right impingement points are denoted by L1-L8 and R1-R8, respectively, and are shown in Figure 7. The plots for unequal left to right flow rates in Figures 9-11 show the double amplitude peak which occurs during the diastolic phase which is absent when the left and right flow rates are equal (see Figure 8). Figure 12 plots WSS versus time for a characteristic point at the left and right impingement regions. Clearly shown in this figure is the double amplitude peak occurring with unequal parent A1 flows and the time between successive peaks as the imbalance between the A1 flows is greater.

\section{Discussion}

The results demonstrate that the flow behavior and hemodynamic stresses at the ACOM bifurcation are highly 


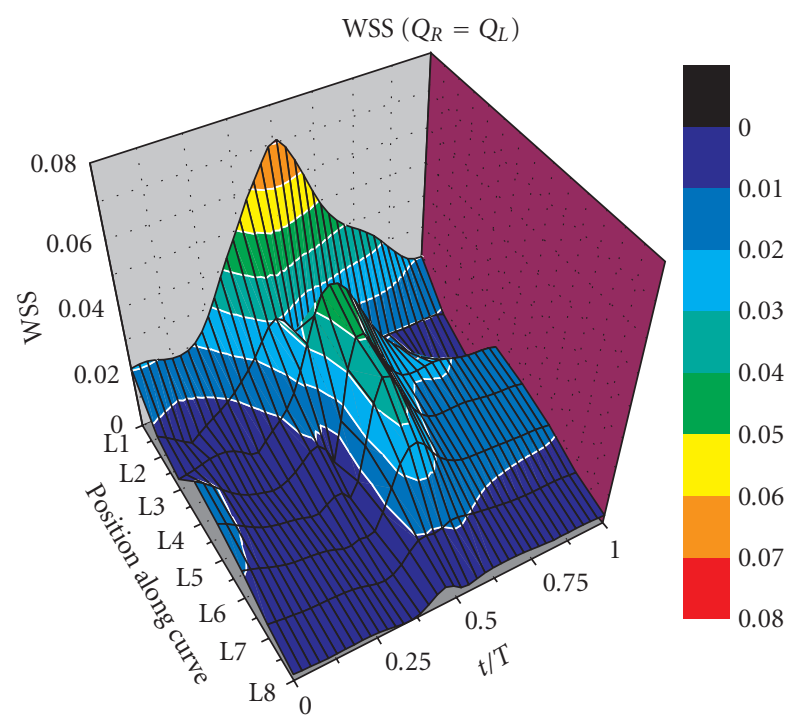

(a)

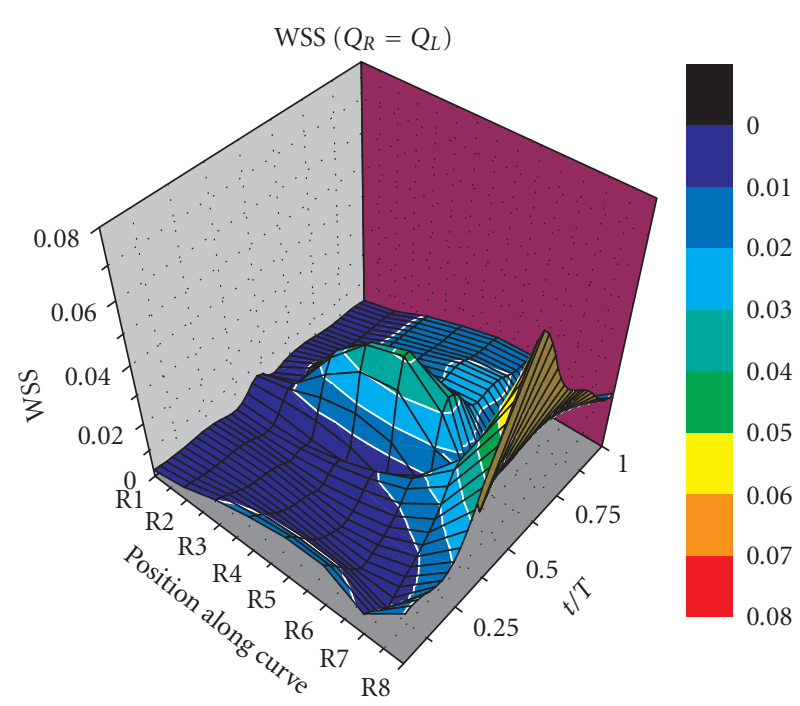

(b)

FIGURE 8: WSS versus position and time for $Q_{\mathrm{R}}=Q_{\mathrm{L}}$ on (a) the left and (b) the right bifurcation regions.

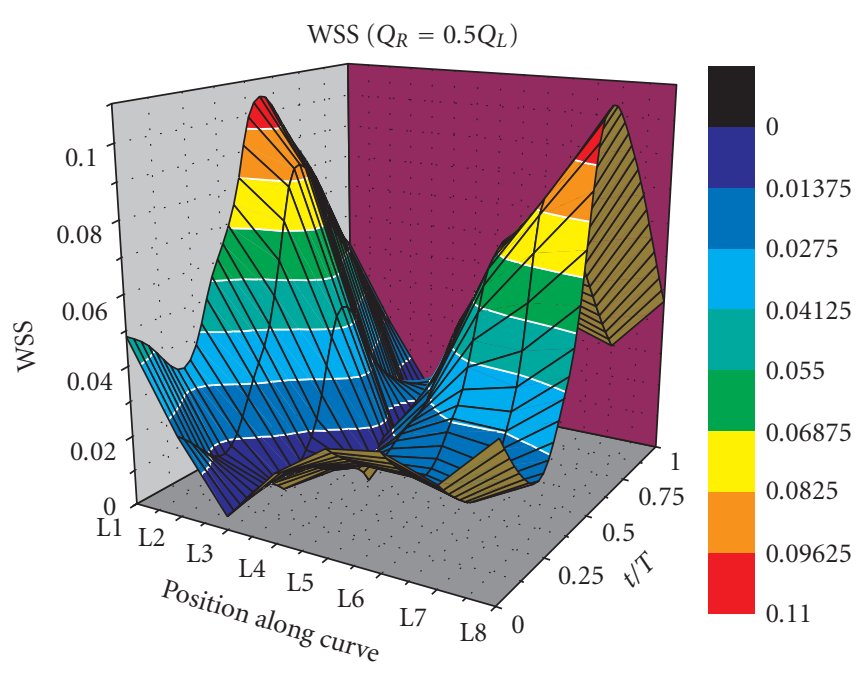

(a)

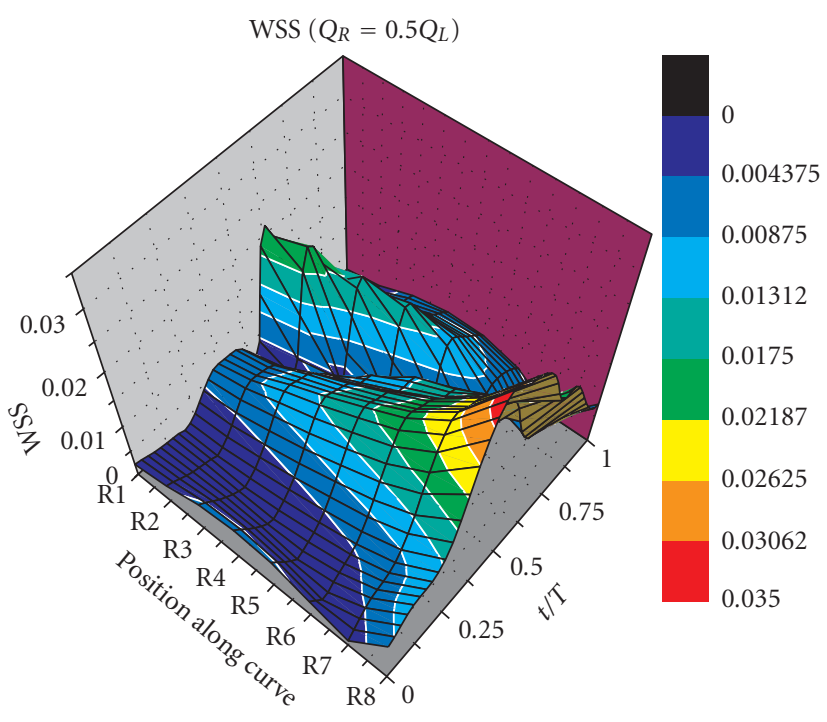

(b)

FIGURE 9: WSS versus position and time for $Q_{R}=0.5 Q_{L}$ on (a) the left and (b) the right bifurcation regions.

dependent on the relative flow rate ratios between the A1ACA parent arteries. Asymmetrical flow conditions at the A1 segments in a symmetrical geometry result in bifurcation of the higher flow parent artery into the A2 and ACOM segments. Cross-flow through the ACOM from the higher flow parent artery is generally unstable at the bifurcation regions especially during diastole. Pulsatility creates a cyclically varying region of flow separation and reversal at those locations which change markedly in amplitude throughout the cycle. Bifurcation regions, where these instabilities occur, are sites of large fluctuations in WSS magnitude and are consistent with the location where aneurysms are predicted to develop.
Unequal flow between left-right A1 segments produces a change in the WSS pattern as compared to symmetrical flow conditions. Both an increase in WSS amplitude at the impingement point and a double amplitude peak at the opposite bifurcation region are observed due to local recirculation effects. The impingement sites of the incoming parent artery flow also shift toward the apex of the bifurcation region along the A2-ACOM adjoining wall where the median muscular layer of the arterial wall is least developed [1]. This leads to greater hemodynamic stresses at locations that are least able to withstand them and a possible mechanism for mechanical deformation from cyclic fatigue stresses. Figures 3-6 also demonstrate that as the impingement site 


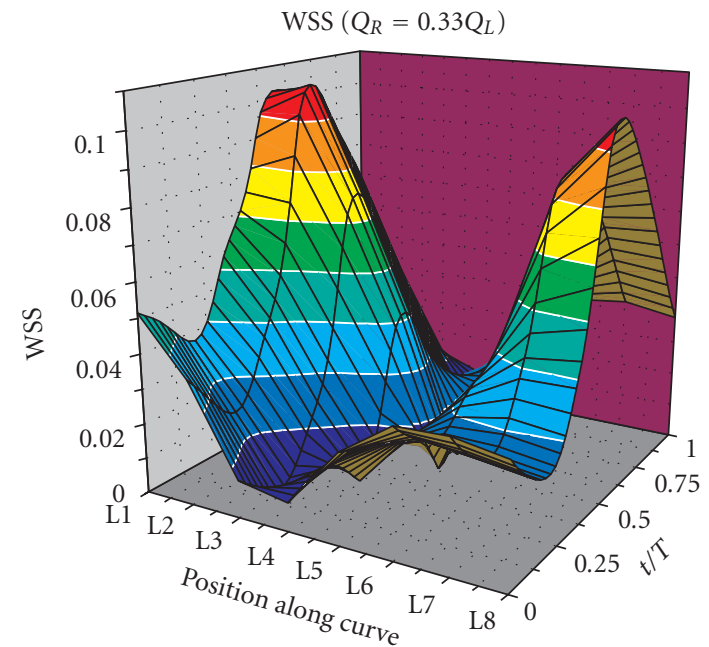

(a)

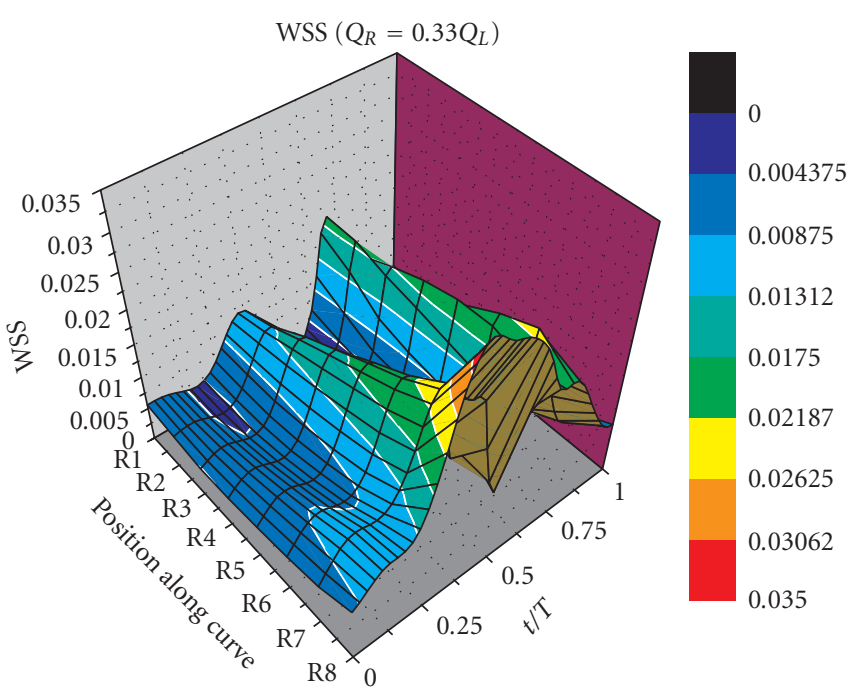

(b)

FIGURE 10: WSS versus position and time for $Q_{R}=0.33 Q_{L}$ on (a) the left and (b) the right bifurcation regions.

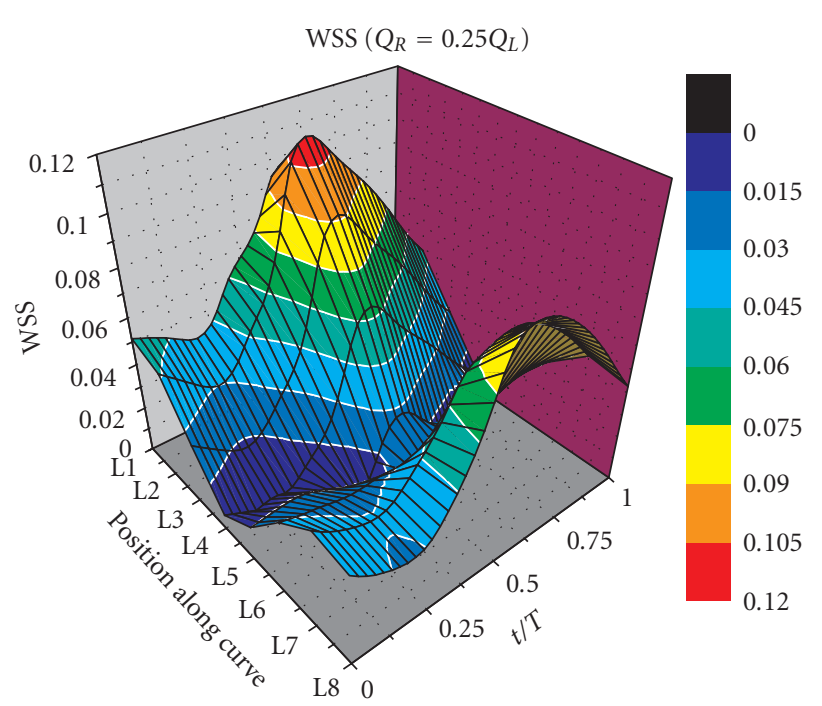

(a)

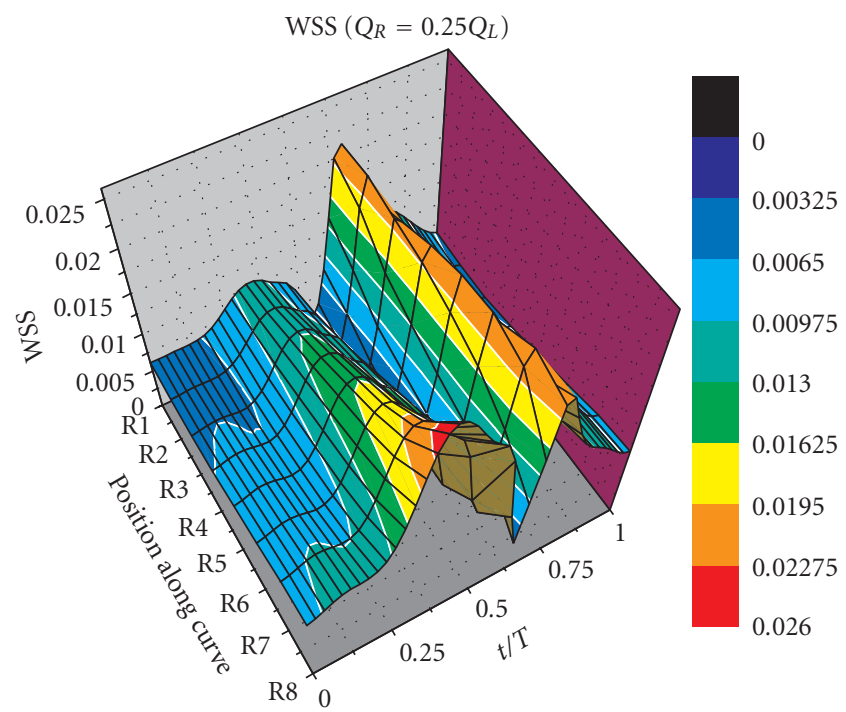

(b)

FIGURE 11: WSS versus position and time for $Q_{R}=0.25 Q_{L}$ on (a) the left and (b) the right bifurcation regions.

from the dominant A1 shifts toward the contralateral ACA with unequal A1 flow rates, the flow impinges at a smaller impingement zone with a more acute angle with respect to the normal of the impingement surface. In addition, the impingement region tends to oscillate along the A2-ACOM adjoining wall with shifting toward the contralateral ACA during systole and vice versa during diastole. By contrast, the case with equal flow rates demonstrates (see Figure 3) that the incoming streams hit at a more oblique angle with respect to the impingement region and oscillate less. Cebral et al. have found that aneurysms that had smaller and changing impingement regions and disturbed flow patterns were associated with rupture. These related findings further strengthen the association of unstable progression of aneurysm growth with large temporal gradient WSS.

Figures 4-6 demonstrate large spatial WSS gradients at the left impingement site where the higher flow parent stream impinges and bifurcates. Spatial gradients from disturbed laminar shear stress from regional flow disturbances have been found to be important local modulators of endothelial cell expression of various growth factors [16]. The plots of the right bifurcation region as seen in Figures $4-6$, and 12 demonstrate that the unsteady effects of flow separation region in unequal flow rates are subject to a double peak in the temporal evolution of WSS. While the magnitude of WSS is decidedly less in the unequal A1 flow 


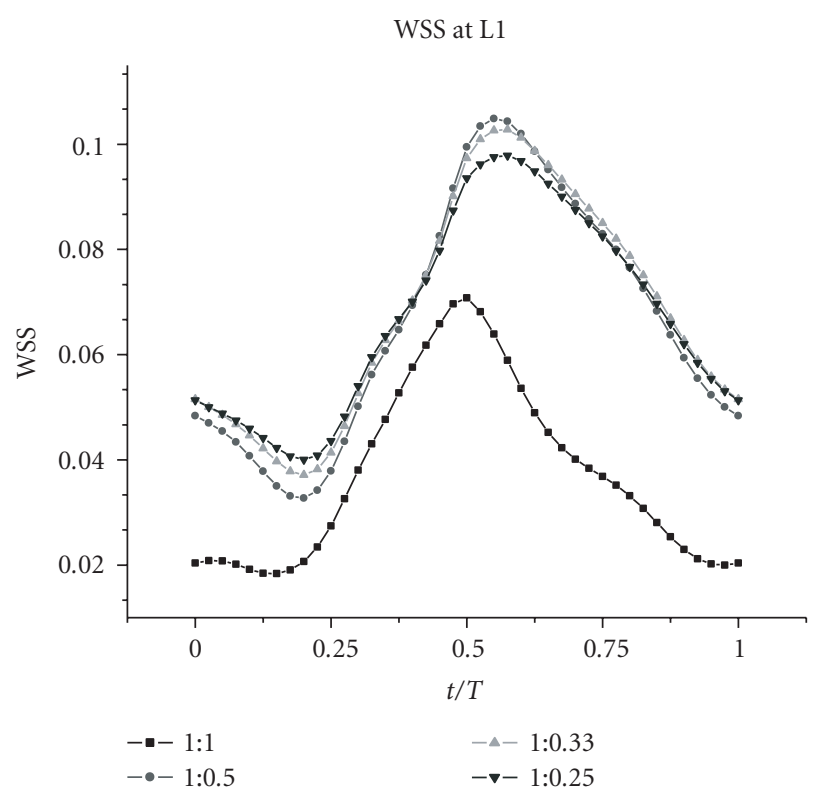

(a)

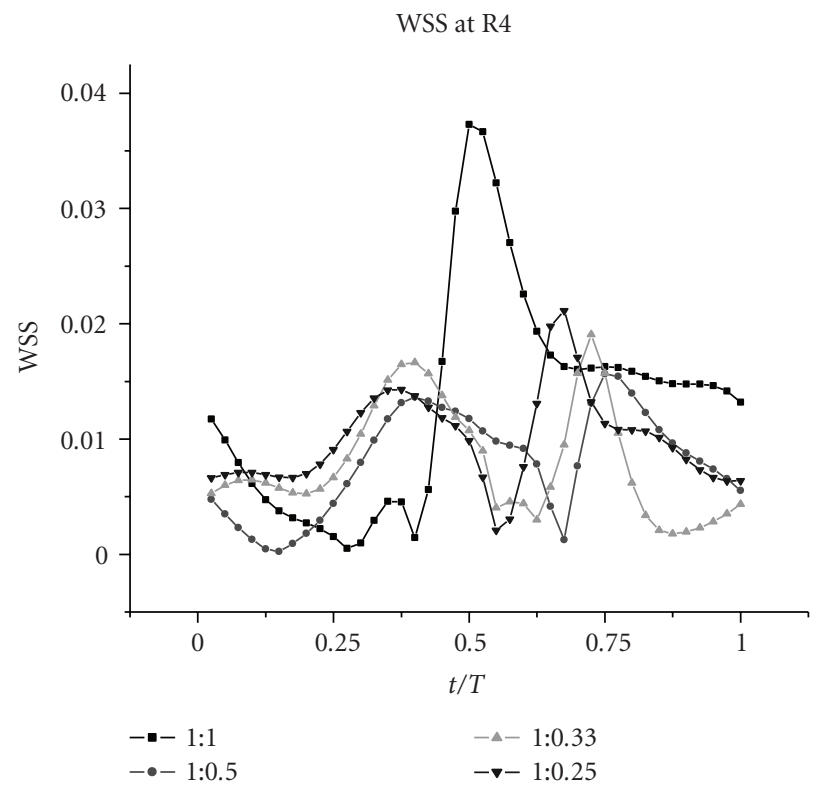

(b)

FIGURE 12: WSS on (a) the left and (b) the right impingement points.

rate condition as compared to equal flow rate conditions, it has been shown that low-amplitude oscillating shear stresses in unsteady flows are an important determining factor in endothelial cell turnover and may be more important than WSS amplitude [17, 18]. Another interesting observation from Figure 12 is that the frequency between the two peaks increases as the imbalance between the incoming A1 inflows is greater. The increased frequency is about $4 \%$ of the total pulsation cycle between the $2: 1$ and $3: 1$ case and increases to $8 \%$ between the $2: 1$ and $4: 1$ studies. These differences reflect the time during the pulsation cycle at which flow separation occurs as the cross-flow navigates through the ACOM segment for the various imbalances in A1 flow. Thus, unequal A1-ACA flow rates induce a change in pattern of hemodynamic stresses which may affect endothelial cells by mechanotransduction in addition to mechanical deformation stresses.

In some cases, flow separation has been shown to be sufficient to cause activation of platelets and to cause freely floating aggregates [19-21]. This suggests that certain regions of unsteady flow may possibly contribute to thromboembolic phenomena. Thus, the ACA-ACOM junction may serve as key centers of platelet aggregates which may release constituents and become a source of emboli. Spontaneous thrombosis and extension of thrombi to bilateral ACAs from an unruptured ACOM aneurysm have been reported in literature and demonstrate that hemodynamic phenomenon in susceptible geometry can lead to cerebrovascular accidents [22].

It is interesting to note that the alteration in hemodynamic stress changes occurs despite the overall reduced flow rate through the anterior cerebral circulation. Unequal flow through the ACAs is a situation which may occur from a number of pathological clinical situations as well as iatrogenic interventions. Intracranial hemorrhage after carotid stenting is a known fatal complication in the postprocedure period [23]. Indeed, the hemodynamic implications of endovascular interventions such as stenotic vessel angioplasty and stenting deserve further investigation. The findings presented may provide some insight into the etiology of wall vessel weakening and perhaps assist in clinical management of patients with ACA-ACOM aneurysms.

The current study is concentrated on the effect of unequal parent ACA flow rates on the hemodynamics of ACOM. By employing a 2D symmetric model, other geometrical factors could be controlled and quick simulation results could be obtained. Even though 2D simulations could be anticipated to reasonably resemble the main characteristics of 3D flows, significant differences between the 2D and 3D simulations are also anticipated to appear in cases of complex geometrical models. The model used in this study employed a high degree of symmetry in an attempt to minimize such 3D confounding factors. More realistic patient-specific 3D models are the subject of future studies.

\section{Conclusions}

Unequal pulsatile flow rates through the A1-ACA arteries result in bifurcation and unstable cross-flow through the ACOM from the higher flow parent artery. There is an increase in WSS magnitude at the impingement site on the same side as the parent A1 segment and a change in position of the maximum WSS related to the left to right flow ratios. The increased hemodynamic stress appears to occur where aneurysms are expected to develop. At the contralateral A2-ACOM adjoining wall, a double peak in the WSS amplitude occurs from local unsteady effects 
arising from flow separation as cross-flow negotiates the ACOM segment. These increased hemodynamic stresses at the bifurcation regions may be a contributing factor to vessel wall weakening where aneurysms often occur.

\section{References}

[1] R. M. K. W. Lee, "Morphology of cerebral arteries," Pharmacology \& Therapeutics, vol. 66, no. 1, pp. 149-173, 1995.

[2] P. F. Davies, C. F. Dewey Jr., S. R. Bussolari, E. J. Gordon, and M. A. Gimbrone Jr., "Influence of hemodynamic forces on vascular endothelial function. In vitro studies of shear stress and pinocytosis in bovine aortic cells," The Journal of Clinical Investigation, vol. 73, no. 4, pp. 1121-1129, 1984.

[3] M. D. Ford, G. R. Stuhne, H. N. Nikolov, et al., "Virtual angiography for visualization and validation of computational models of aneurysm hemodynamics," IEEE Transactions on Medical Imaging, vol. 24, no. 12, pp. 1586-1592, 2005.

[4] X. He, P. Venugopal, J. R. Cebral, H. Schmitt, and D. J. Valentino, "Reproducibility of brain hemodynamic simulations: an inter-solver comparison," in Medical Imaging 2006: Physiology, Function, and Structure from Medical Images, vol. 6143 of Proceedings of SPIE, pp. 1-12, San Diego, Calif, USA, February 2006.

[5] J. R. Cebral, M. A. Castro, J. E. Burgess, R. S. Pergolizzi, M. J. Sheridan, and C. M. Putman, "Characterization of cerebral aneurysms for assessing risk of rupture by using patientspecific computational hemodynamics models," American Journal of Neuroradiology, vol. 26, no. 10, pp. 2550-2559, 2005.

[6] D. N. Ku, "Blood flow in arteries," Annual Review of Fluid Mechanics, vol. 29, pp. 399-434, 1997.

[7] D. N. Ku, D. P. Giddens, C. K. Zarins, and S. Glagov, "Pulsatile flow and atherosclerosis in the human carotid bifurcation. Positive correlation between plaque location and low and oscillating shear stress," Arteriosclerosis, vol. 5, no. 3, pp. 293 302, 1985.

[8] S. A. Berger and L.-D. Jou, "Flows in stenotic vessels," Annual Review of Fluid Mechanics, vol. 32, pp. 347-382, 2000.

[9] C. A. Taylor, T. J. R. Hughes, and C. K. Zarins, "Finite element modeling of blood flow in arteries," Computer Methods in Applied Mechanics and Engineering, vol. 158, no. 1-2, pp. 155196, 1998.

[10] R. Kwak, T. Ohi, H. Niizuma, and J. Suzuki, "Afferent artery and the site of neck of anterior communicating aneurysms," Surgical Neurology, vol. 13, no. 3, pp. 221-223, 1980.

[11] http://www.solidworks.com.

[12] F. Cassot, V. Vergeur, P. Bossuet, B. Hillen, M. Zagzoule, and J.-P. Marc-Vergnes, "Effects of anterior communicating artery diameter on cerebral hemodynamics in internal carotid artery disease: a model study," Circulation, vol. 92, no. 10, pp. 31223131, 1995.

[13] M. A. Stefani, F. L. Schneider, A. C. H. Marrone, A. G. Severino, A. P. Jackowski, and M. C. Wallace, "Anatomic variations of anterior cerebral artery cortical branches," Clinical Anatomy, vol. 13, no. 4, pp. 231-236, 2000.

[14] G. Batchelor, An Introduction to Fluid Dynamics, Cambridge University Press, Cambridge, UK, 2000.

[15] http://www.opencfd.co.uk/openfoam.

[16] T. Nagel, N. Resnick, C. F. Dewey Jr., and M. A. Gimbrone Jr., "Vascular endothelial cells respond to spatial gradients in fluid shear stress by enhanced activation of transcription factors," Arteriosclerosis, Thrombosis, and Vascular Biology, vol. 19, no. 8, pp. 1825-1834, 1999.
[17] P. F. Davies, A. Remuzzi, E. J. Gordon, C. F. Dewey Jr., and M. A. Gimbrone Jr., "Turbulent fluid shear stress induces vascular endothelial cell turnover in vitro," Proceedings of the National Academy of Sciences of the United States of America, vol. 83, no. 7, pp. 2114-2117, 1986.

[18] E. Sho, H. Nanjo, M. Sho, et al., "Arterial enlargement, tortuosity, and intimal thickening in response to sequential exposure to high and low wall shear stress," Journal of Vascular Surgery, vol. 39, no. 3, pp. 601-612, 2004.

[19] W. A. Morton, E. M. Parmentier, and H. E. Petschek, "Study of aggregate formation in region of separated blood flow," Thrombosis et Diathesis Haemorrhagica, vol. 34, no. 3, pp. 840854, 1975.

[20] S. Einav and D. Bluestein, "Dynamics of blood flow and platelet transport in pathological vessels," Annals of the New York Academy of Sciences, vol. 1015, pp. 351-366, 2004.

[21] O. A. el-Masry, I. A. Feuerstein, and G. F. Round, "Experimental evaluation of streamline patterns and separated flows in a series of branching vessels with implications for atherosclerosis and thrombosis," Circulation Research, vol. 43, no. 4, pp. 608-618, 1978.

[22] R. D. Brownlee, B. I. Tranmer, R. J. Sevick, G. Karmy, and B. J. Curry, "Spontaneous thrombosis of an unruptured anterior communicating artery aneurysm: an unusual cause of ischemic stroke," Stroke, vol. 26, no. 10, pp. 1945-1949, 1995.

[23] W. F. Morrish, S. Grahovac, A. Douen, et al., "Intracranial hemorrhage after stenting and angioplasty of extracranial carotid stenosis," American Journal of Neuroradiology, vol. 21, no. 10, pp. 1911-1916, 2000. 

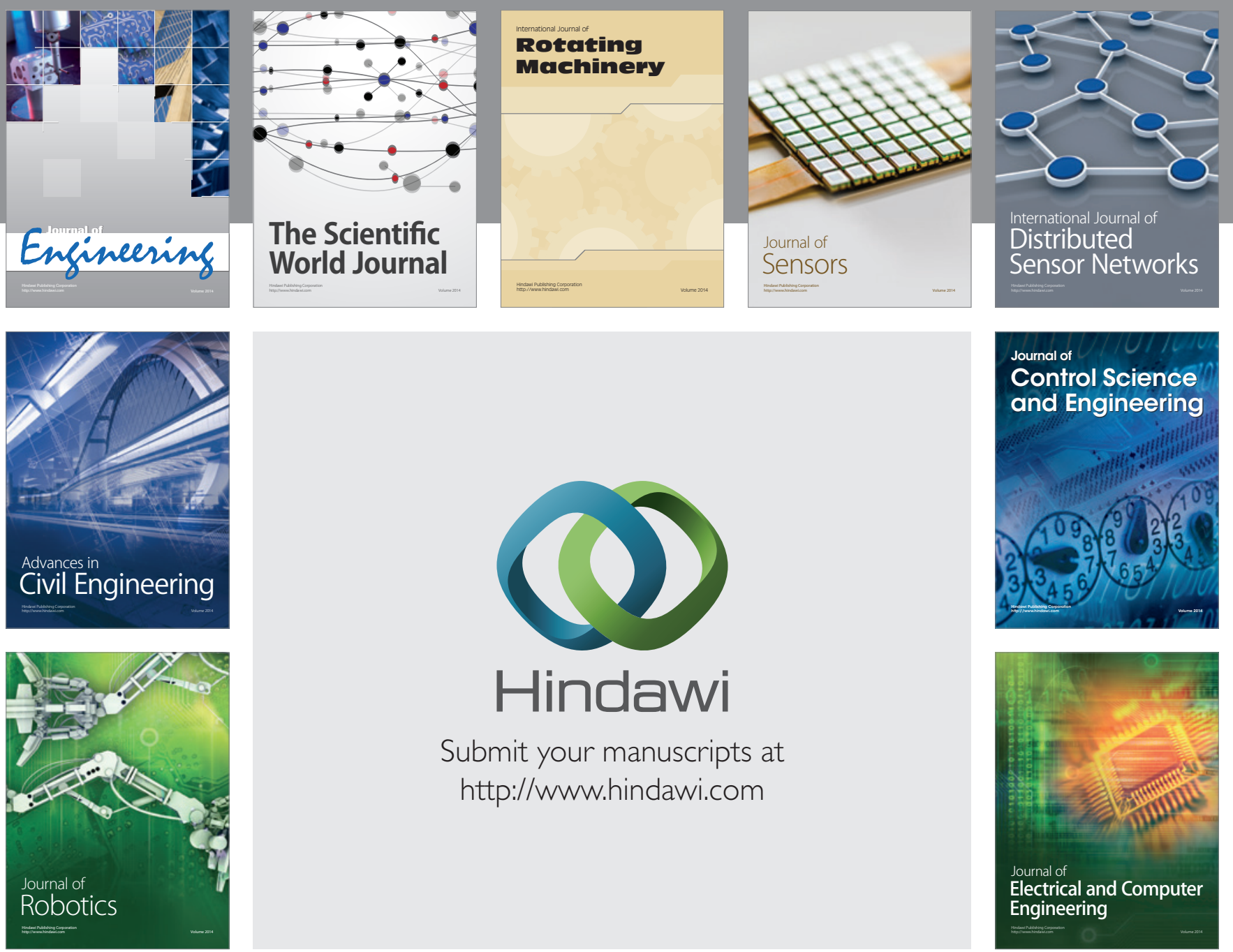

Submit your manuscripts at

http://www.hindawi.com
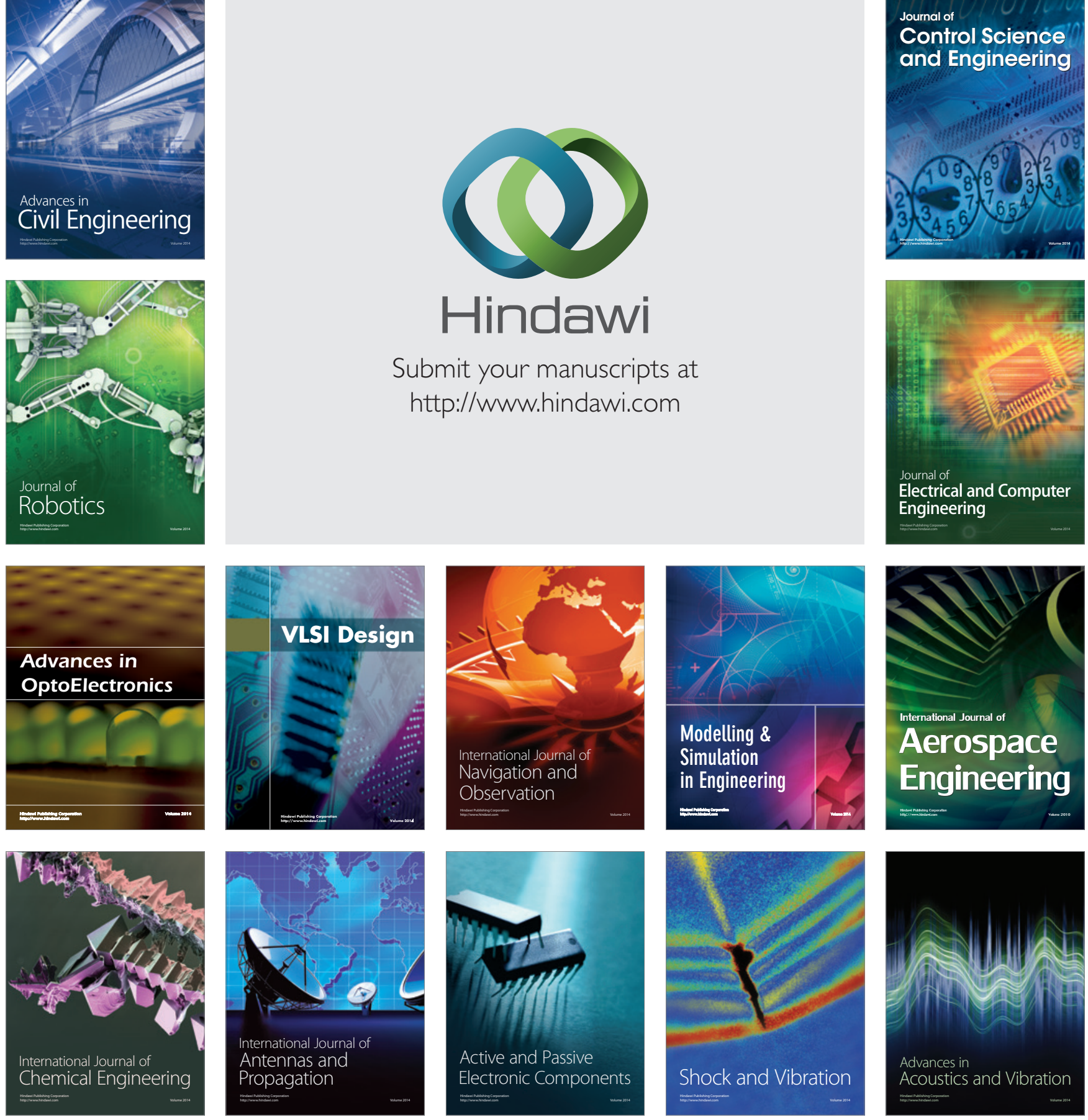